\title{
Predictive factors for sepsis by carbapenem resistant Gram-negative bacilli in adult critical patients in Rio de Janeiro: a case- case-control design in a prospective cohort study
}

Elisangela M. Lima ${ }^{2,3}$, Patrícia A. Cid², Debora S. Beck², Luiz Henrique Z. Pinheiro², João Pedro S. Tonhá1, Marcio Z. O. Alves ${ }^{3}$, Newton D. Lourenço ${ }^{3}$, Roberto Q. Santos³, Marise D. Asensi², José Aurélio Marques³, Carolina S. Bandeira', Caio Augusto S. Rodrigues ${ }^{3}$, Saint Clair S. Gomes Junior ${ }^{4}$, Marisa Z. R. Gomes ${ }^{1,2,3^{*}}$ (D) and the Nucleus of Hospital Research study collaborators

\begin{abstract}
Background: Studies have investigated risk factors for infections by specific species of carbapenem-resistant Gramnegative bacilli (CR-GNB), but few considered the group of GNB species and most of them were performed in the setting of bacteremia or hospital infection. This study was implemented to identify risk factors for sepsis by CR- and carbapenem-susceptible (CS) GNB in intensive care unit (ICU) patients to improve management strategies for CRGNB sepsis.
\end{abstract}

Methods: We developed a case-case-control study from a prospective cohort of patients with systemic inflammatory response syndrome (SIRS), sepsis-2 or sepsis-3 criteria in which blood and other sample cultures were collected and antimicrobial therapy was instituted, in an adult clinical-surgical ICU, at tertiary public hospital in Rio de Janeiro, from August 2015 through March 2017.

(Continued on next page)

\footnotetext{
* Correspondence: marisargomes@ioc.fiocruz.br; marisargomes@hotmail.com

Partly presented in the 13th World Congress of Intensive and Critical Care Medicine, Rio de Janeiro, Brazil, 2017. Abstract Number EP-099.

${ }^{1}$ Laboratório de Genética Molecular de Microrganismos, Instituto Oswaldo Cruz, Oswaldo Cruz Foundation (IOC/FIOCRUZ), Rio de Janeiro, RJ, Brazil

${ }^{3}$ Hospital Federal dos Servidores do Estado (HFSE), Ministry of Health, Rio de Janeiro, RJ, Brazil

Full list of author information is available at the end of the article
}

(c) The Author(s). 2020 Open Access This article is licensed under a Creative Commons Attribution 4.0 International License, which permits use, sharing, adaptation, distribution and reproduction in any medium or format, as long as you give appropriate credit to the original author(s) and the source, provide a link to the Creative Commons licence, and indicate if changes were made. The images or other third party material in this article are included in the article's Creative Commons licence, unless indicated otherwise in a credit line to the material. If material is not included in the article's Creative Commons licence and your intended use is not permitted by statutory regulation or exceeds the permitted use, you will need to obtain permission directly from the copyright holder. To view a copy of this licence, visit http://creativecommons.org/licenses/by/4.0/ The Creative Commons Public Domain Dedication waiver (http://creativecommons.org/publicdomain/zero/1.0/) applies to the data made available in this article, unless otherwise stated in a credit line to the data. 
(Continued from previous page)

Results: Among the total of 629 ICU admissions followed by 7797 patient-days, after applying inclusion and exclusion criteria we identified 184 patients who developed recurrent or single hospital-acquired sepsis. More than $90 \%$ of all evaluable cases of sepsis and $87 \%$ of control group fulfilled the modified sepsis-3 definition. Nonfermenting bacilli and ventilator-associated pneumonia predominated as etiology and source of CR-GNB sepsis. While Enterobacteriaceae and intra-abdominal surgical site plus urinary-tract infections prevailed in CS-GNB than CR-GNB sepsis. Carbapenemase production was estimated in 76\% of CR-GNB isolates. Multivariate logistic regression analysis revealed previous infection (mostly hospital-acquired bacterial infection or sepsis) $(\mathrm{OR}=4.28$; $95 \% \mathrm{Cl} 1.77-$ 10.35), mechanical ventilation $(O R=4.21 ; 95 \% \mathrm{Cl} 1.17-15.18)$, carbapenem use $(O R=3.42 ; 95 \% \mathrm{Cl} 1.37-8.52)$ and length of hospital stay $(\mathrm{OR}=1.03 ; 95 \% \mathrm{Cl} 1.01-1.05)$ as independent risk factors for sepsis by CR-GNB. While ICU readmission $(\mathrm{OR}=6.92 ; 95 \% \mathrm{Cl} 1.72-27.78)$ and nosocomial diarrhea $(\mathrm{OR}=5.32 ; 95 \% \mathrm{Cl} 1.07-26.45)$ were factors associated with CS-GNB sepsis.

Conclusions: The investigation of recurrent and not only bacteremic episodes of sepsis was the differential of this study. The results are in agreement with the basic information in the literature. This may help improve management strategies and future studies on sepsis by CR-GNB.

Keywords: Sepsis, Gram-negative bacilli, Antimicrobial resistance, Risk factors, Hospital infection, Polymerase chain reaction

\section{Background}

Sepsis is a leading cause of morbidity and mortality, during and after hospitalization in intensive care units (ICU) [1, 2]. Carbapenem-resistant (CR) Acinetobacter baumannii, Pseudomonas aeruginosa and Enterobacteriaceae have become major pathogens, especially in ICUs, implicated in healthcare-associated sepsis, causing prolonged hospitalization, high mortality, and increased costs [3-6].

Several studies in the literature have investigated risk factors for infections by specific species of CR-Gramnegative bacilli (GNB) [7-17], but few considered all detected GNB species $[18,19]$, and most of them were in the context of nosocomial infection [9, 10, 12, 15-17] or bacteremia $[8,11,18,19]$.

The empirical therapy of sepsis should be started within the first hour of presumed diagnosis, at a time when the clinic-epidemiological characteristics remain as the only determinants of a patient at greater risk.

Given these facts, we performed a case-case-control study to investigate predictive factors for sepsis by CR- and carbapenem-susceptible (CS) GNB in adult patients from a Brazilian public ICU. Our goal is to develop and validate a predictive score to identify patients at higher risk for CR-GNB sepsis in future studies.

\section{Methods}

\section{Patients, setting and study design}

The study followed a case-control design from a prospective cohort of patients with SIRS, sepsis-2 or sepsis3 criteria in which blood and other samples' cultures were collected and antimicrobial therapy was instituted, for two or more days, in an adult clinical-surgical ICU, at a tertiary public hospital in Rio de Janeiro, Brazil, from August 2015 through March 2017. This study was approved by the institutional ethics committee and followed the Declaration of Helsinki and its later amendments. The study followed the STROBE recommendations for observational cohort studies (STROBE list in Additional file 1: Appendix S4) [20].

We evaluated all patients` clinical and surveillance samples cultured during the episodes, throughout the ICU stay, and the follow-up period of 30 days following the end of sepsis treatment. Surveillance cultures were not used to assess patient inclusion or exclusion in the study. Patients were not matched by any variable, considering the homogeneity of this population.

We followed all detected sepsis episodes during the ICU stay and follow-up period. Patients with more than one episode were aleatory selected in each group in such a manner that patients who presented CR-GNB sepsis were selected as case 1, patients who had CS-GNB sepsis episode were selected as case 2 and those with unknown sepsis or due to other etiologies than GNB were selected as control group. Cases and controls entered the study once and were monitored closely during the follow-up period.

We excluded patients younger than 18 years old, those with sepsis acquired in the community or associated with another healthcare institution, those who refused to sign the consent form and those suffering from polymicrobial sepsis by GNB and non GNB agents. In addition, we excluded patients initially enrolled in the control or CS-GNB case group that evolved respectively with CS-GNB or CR-GNB infection after discharge from ICU and during the followup period. 
Table 1 Clinical characteristic investigated for association with CR-GNB sepsis (Case group 1) in an adult clinical-surgical ICU

\begin{tabular}{|c|c|c|c|c|c|}
\hline \multirow[t]{2}{*}{ Predictive Factors } & \multicolumn{2}{|l|}{$\underline{\text { Univariate analysis }}$} & \multirow[t]{2}{*}{ Odds ratio } & \multirow[t]{2}{*}{$95 \% \mathrm{Cl}$} & \multirow[t]{2}{*}{$\overline{P^{t} \text { valor }}$} \\
\hline & CR-GNB $(n=60)$ & Controls $(n=94)$ & & & \\
\hline \multicolumn{6}{|l|}{ Demographic data } \\
\hline Age in years, median (range) & $62(23-91)$ & $62(19-92)$ & 1.00 & $0.98-1.02$ & 0.95 \\
\hline Male sex, n (\%) & $36(60)$ & $46(49)$ & 1.57 & $0.81-3.02$ & 0.18 \\
\hline \multicolumn{6}{|l|}{ Comorbidities $^{a}, \mathrm{n}(\%)$} \\
\hline Diabetes mellitus ${ }^{\mathrm{b}}$ & $21(35)$ & $35(37)$ & 0.91 & $0.46-1.78$ & 0.78 \\
\hline Renal failure ${ }^{c}$ & $30(50)$ & $38(40)$ & 1.47 & $0.77-2.83$ & 0.25 \\
\hline Hemodialysis $^{d}$ & $22(37)$ & $18(19)$ & 2.44 & $1.17-5.10$ & 0.02 \\
\hline Chronic liver disease $e^{e}$ & $2(3)$ & $9(10)$ & 0.33 & $0.07-1.56$ & 0.16 \\
\hline Immunossupressive condition $^{f}$ & $9(15)$ & $11(12)$ & 1.33 & $0.52-3.43$ & 0.55 \\
\hline Gastrointestinal disease & $16(27)$ & $18(19)$ & 1.54 & $0.71-3.31$ & 0.27 \\
\hline Geniturinary disease & $5(8)$ & $9(10)$ & 0.86 & $0.27-2.70$ & 0.79 \\
\hline Pulmonary disease & $10(17)$ & $14(15)$ & 1.14 & $0.47-2.77$ & 0.77 \\
\hline AIDS or chronic infectious disease & $3(5)$ & $3(3)$ & 1.60 & $0.31-8.18$ & 0.56 \\
\hline Surgery ${ }^{9}$ & $26(43)$ & $46(49)$ & 0.80 & $0.42-1.53$ & 0.50 \\
\hline Infection/Colonization by CR-GNB & $19(32)$ & $7(7)$ & 5.76 & $2.24-14.79$ & $<0.001$ \\
\hline Nosocomial diarrhea $^{\text {h }}$ & $17(28)$ & $6(6)$ & 5,80 & $2.13-15.75$ & 0.001 \\
\hline Neutropenia' & $5(8)$ & $4(4)$ & 2.05 & $0.53-7.94$ & 0.30 \\
\hline Neoplasm & $23(38)$ & $28(30)$ & 1.47 & $0.74-2.90$ & 0.27 \\
\hline Infection & $46(77)$ & $31(33)$ & 6.68 & $3.20-13.95$ & $<0.001$ \\
\hline Prior ICU hospitalization, n (\%) & $7(12)$ & $5(5)$ & 2.35 & $0.71-7.78$ & 0.26 \\
\hline \multicolumn{6}{|l|}{ Length of hospital stay (in days) } \\
\hline Median (range) & $26.5(1-375)$ & $10(0-143)$ & 1.04 & $1.02-1.06$ & $<0.001$ \\
\hline \multicolumn{6}{|l|}{ ICU hospitalization reason, n (\%) } \\
\hline Elective or emergency surgery & $10(17)$ & $28(30)$ & 0.47 & $0.21-1.06$ & 0.07 \\
\hline Respiratory tract disease & $16(27)$ & $14(15)$ & 2.08 & $0.93-4.65$ & 0.08 \\
\hline Cardiovascular disease & $6(10)$ & $8(9)$ & 1.19 & $0.39-3.63$ & 0.75 \\
\hline Neurological disease & $2(3)$ & $11(12)$ & 0.26 & $0.06-1.22$ & 0.09 \\
\hline Gastrointestinal disease & $3(5)$ & $5(5)$ & 0.94 & $0.22-4.07$ & 0.93 \\
\hline Renal pathology & $5(8)$ & $11(12)$ & 0.69 & $0.23-2.08$ & 0.51 \\
\hline Sepsis & $31(52)$ & $30(32)$ & 2.40 & $1.23-4.68$ & 0.01 \\
\hline Sepsis shock & $23(38)$ & $27(39)$ & 1.63 & $0.82-3.24$ & 0.17 \\
\hline \multicolumn{6}{|l|}{ Total SOFA score at ICU admission, } \\
\hline Median (range) & $6(1-17)$ & $6(0-17)$ & 1.06 & $0.98-1.15$ & 0.16 \\
\hline SAPs 3 score $^{k}$, median (range) & $65(30-103)$ & $64(29-105)$ & 1.01 & $0.99-1.03$ & 0.39 \\
\hline \multicolumn{6}{|l|}{ Invasive devices, n (\%) } \\
\hline Mechanical ventilation & $56(93)$ & $58(62)$ & 8.69 & $2.90-26.01$ & $<0.001$ \\
\hline Central vascular catheter & $60(100)$ & $81(86)$ & $\ldots$ & $\ldots$ & 0.003 \\
\hline Urinary catheter & $55(92)$ & $72(76)$ & 3.16 & $1.12-8.92$ & 0.03 \\
\hline \multicolumn{6}{|l|}{ Previous use of antimicrobials, n (\%) } \\
\hline Aminoglycosides' & $18(30)$ & $7(7)$ & 5.33 & $2.07-13.74$ & 0.001 \\
\hline Cephalosporins, 3rd and $4 \mathrm{rd}$ generations ${ }^{\mathrm{m}}$ & $8(13)$ & $16(17)$ & 0.75 & $0.30-1.88$ & 0.54 \\
\hline Carbapenems $^{\mathrm{n}}$ & $48(80)$ & $32(34)$ & 7.75 & $3.61-16.62$ & $<0.001$ \\
\hline Glycopeptides $^{\circ}$, linezolid and tigecycline & $45(75)$ & $35(37)$ & 5.06 & $2.47-10.37$ & $<0.001$ \\
\hline
\end{tabular}


Table 1 Clinical characteristic investigated for association with CR-GNB sepsis (Case group 1) in an adult clinical-surgical ICU (Continued)

\begin{tabular}{|c|c|c|c|c|c|}
\hline \multirow[t]{2}{*}{ Predictive Factors } & \multicolumn{2}{|c|}{ Univariate analysis } & \multirow[t]{2}{*}{ Odds ratio } & \multirow[t]{2}{*}{$95 \% \mathrm{Cl}$} & \multirow[t]{2}{*}{$P^{t}$ valor } \\
\hline & CR-GNB $(n=60)$ & Controls $(n=94)$ & & & \\
\hline Fluoroquinolones $^{p}$ & $14(23)$ & $15(16)$ & 1.60 & $0.71-3.62$ & 0.26 \\
\hline Metronidazole & $11(18)$ & $13(14)$ & 1.40 & $0.58-3.37$ & 0.46 \\
\hline Piperacilin-tazobactam & $30(50)$ & $21(22)$ & 3.48 & $1.72-7.01$ & $<0.001$ \\
\hline Polymyxins $^{q}$ & $22(37)$ & $9(10)$ & 5.47 & $2.30-12.98$ & $<0.001$ \\
\hline ATB with action for anaerobes ${ }^{r}$ & $56(93)$ & $59(63)$ & 8.31 & $2.77-24.88$ & $<0.001$ \\
\hline Antifungal agents ${ }^{5}$ & $26(43)$ & $13(14)$ & 4.77 & $2.19-10.36$ & $<0.001$ \\
\hline
\end{tabular}

Abbreviations: AIDS Acquired Immunodeficiency Syndrome, ATB antibiotic agents, CI Confidence interval, CR-GNB Carbapenem resistant Gram-negative bacilli, ICU Intensive Care Unit

${ }^{\text {a }}$ Prior comorbidities or conditions to investigated sepsis episode

${ }^{b}$ Diagnosis of diabetes mellitus requiring oral or injectable hypoglycemic drug

${ }^{c}$ Creatinine clearance $<30 \mathrm{cc} / \mathrm{min}$

${ }^{\mathrm{d}}$ Required in the last 90 days

eLaboratory clinical evidence

f Prednisone $>10 \mathrm{mg}$ for more than 50 days, corticosteroid for $>7$ days or immunomodulatory agents (examples: monoclonal agents, methotrexate)

In the last 30 days

${ }^{\mathrm{h}}$ Nosocomial diarrhea ( 3 or more daily episodes of stool for 2 or more days)

iGranulocytes $<500$ cells $/ \mathrm{mm}^{3}$

jSequential Organ Failure Assessment score

ksimplified Acute Physiology Score III

Amikacin and gentamicin

${ }^{\mathrm{m}}$ Ceftriaxone, ceftazidime and cefepime

nErtapenem, imipenem-cilastatin and meropenem

${ }^{\circ}$ Daptomycin, teicoplanin and vancomycin

${ }^{\mathrm{P} C i p r o f l o x a c i n}$, levofloxacin, moxifloxacin

qPolymyxin B and colistin

'Antibacterial agents with action for anaerobes - Amoxicilin-clavulanate, ampicillin-sulbactam, piperacilin-tazobactam, clindamycin, ertapenem, imipenem, meropenem and metronidazole

${ }^{5}$ Amphotericin B family (standard, lipid complex or liposomal Amphotericin), echinocandins and azoles

tPearson's chi-square test or Fisher's exact test or Mann-Whitney-Wilcoxon $U$ test, as required, and considering statistically significant $p<0.05$

The variables investigated as predictive factors were studied during the period of hospitalization prior to sepsis episode for both cases and controls. We investigated demographics and comorbidities, length of hospital stays, prior ICU hospitalization, reasons for ICU admission, simplified acute physiology score (SAPS-3) and sequential organ failure assessment (SOFA) at baseline, previous use of invasive devices and antimicrobials. The variables and their definitions are described in Tables 1 and 2. The information was collected from multiple sources including hospital records, hospital laboratory system, radiological records, hospital infection control committee daily surveillance, ICU staff daily clinical round records, and data entered daily into the ICU Epimed System. We collected and managed study data using REDCap electronic data capture tools hosted at Instituto Oswaldo Cruz, Fundação Oswaldo Cruz (IOC/ FIOCRUZ). The study formularies with the investigated variables are in Additional file 1: Appendices S1, S2 and S3. To avoid potential bias, all data collected were standardized and monitored throughout the study.

\section{Definitions}

Recurrent sepsis was defined as a new episode of sepsis developing after resolution of clinical and laboratory parameters of sepsis, or the recrudescence of sepsis with the evidence of new etiology by cultures during ICU stay. An adaptation of Singer et al. (2016) [21] sepsis-3 criteria was used retrospectively as follows: delta SOFA $\geq 2$ between SOFA scores measured on two calendar days between the period of $72 \mathrm{~h}$ that preceded to $24 \mathrm{~h}$ that succeeded the date of initial blood culture, and on the ICU admission date; and qSOFA applied in patients without mechanical ventilation or sedation within $72 \mathrm{~h}$ before and $24 \mathrm{~h}$ after the date of blood culture.

Post-hoc analysis was performed by two research infectious disease physician investigators to review all clinical, radiological, and microbiological data. We reviewed the evidence of sepsis-2 [22] and - 3 [21], and of the infectious source. We classified the plausibility of infectious source as definitive, probable, possible or undetermined, according to Klouwenberg et al. (2013) criteria [23], adapted to include the updated Centers for Disease Control and Prevention definitions [24].

\section{Microbiological methods}

Blood cultures (aerobic and anaerobic) were processed using the BD BACTEC ${ }^{\mathrm{mx}}$ system (Becton Dickinson, Sparks, MD, EUA), according to the routine of hospital microbiology laboratory. Identification and antibiotic 
Table 2 Clinical characteristic investigated for association with CS-GNB sepsis (Case group 2) in an adult clinical-surgical ICU

\begin{tabular}{|c|c|c|c|c|c|}
\hline \multirow[t]{2}{*}{ Predictive Factors } & \multicolumn{2}{|c|}{ Univariate analysis } & \multirow[t]{2}{*}{ Odds ratio } & \multirow[t]{2}{*}{$95 \% \mathrm{Cl}$} & \multirow[t]{2}{*}{$p^{t}$ valor } \\
\hline & $\overline{C S-G N B}(n=30)$ & Controls $(n=94)$ & & & \\
\hline \multicolumn{6}{|l|}{ Demographic data } \\
\hline Age in years, median (range) & $66(27-82)$ & $62(19-92)$ & 1.01 & $0.98-1.04$ & 0.46 \\
\hline Male sex, n (\%) & $9(30)$ & $46(49)$ & 2.24 & $0.93-5.39$ & 0.07 \\
\hline \multicolumn{6}{|l|}{ Comorbidities $^{a}, \mathrm{n}(\%)$} \\
\hline Diabetes mellitus ${ }^{\mathrm{b}}$ & $15(50)$ & $35(37)$ & 1.69 & $0.74-3.86$ & 0.22 \\
\hline Renal failure ${ }^{c}$ & $8(27)$ & $38(40)$ & 0.54 & $0.22-1.33$ & 0.18 \\
\hline Hemodialysis $^{d}$ & $3(10)$ & $18(19)$ & 0.47 & $0.13-1.72$ & 0.25 \\
\hline Chronic liver disease $\mathrm{e}^{\mathrm{e}}$ & $2(7)$ & $9(10)$ & 0.67 & $0.14-3.31$ & 0.63 \\
\hline Immunossupressive condition $^{f}$ & $6(20)$ & $11(12)$ & 1.89 & $0.63-5.63$ & 0.26 \\
\hline Gastrointestinal disease & $9(30)$ & $18(19)$ & 1.81 & $0.71-4.61$ & 0.21 \\
\hline Geniturinary disease & $3(10)$ & $9(10)$ & 1.05 & $0.27-4.16$ & 0.95 \\
\hline Pulmonary disease & $7(23)$ & $14(15)$ & 1.74 & $0.63-4.82$ & 0.29 \\
\hline AIDS or chronic infectious disease & $2(7)$ & $3(3)$ & 2.17 & $0.35-13.62$ & 0.41 \\
\hline Surgery ${ }^{9}$ & $19(63)$ & $46(49)$ & 1.80 & $0.77-4.20$ & 0.17 \\
\hline Infection/Colonization by CR-GNB & $5(17)$ & $7(7)$ & 2.49 & $0.73-8.51$ & 0.15 \\
\hline Nosocomial diarrhea ${ }^{\text {h }}$ & $5(17)$ & $6(6)$ & 2.93 & $0.83-10.42$ & 0.10 \\
\hline Neutropenia ${ }^{i}$ & $1(3)$ & $4(4)$ & 0.78 & $0.08-7.22$ & 0.82 \\
\hline Neoplasm & $17(57)$ & $28(30)$ & 3.08 & $1.32-7.19$ & 0.009 \\
\hline Infection & $10(33)$ & $31(33)$ & 0.92 & $0.39-2.21$ & 0.86 \\
\hline Prior ICU hospitalization, n (\%) & $9(30)$ & $5(5)$ & 7.63 & $2.32-25.13$ & 0.001 \\
\hline \multicolumn{6}{|l|}{ Length of hospital stay (in days) } \\
\hline Median (range) & $15(0-142)$ & $10(0-143)$ & 1.01 & $0.99-1.03$ & 0.16 \\
\hline \multicolumn{6}{|l|}{ ICU hospitalization reason, n (\%) } \\
\hline Elective or emergency surgery & $10(33)$ & $28(30)$ & 1.18 & $0.49-2.84$ & 0.71 \\
\hline Respiratory tract disease & $5(17)$ & $14(15)$ & 1.14 & $0.38-3.49$ & 0.82 \\
\hline Cardiovascular disease & $1(3)$ & $8(9)$ & 0.37 & $0.04-3.09$ & 0.36 \\
\hline Neurological disease & $3(10)$ & $11(12)$ & 0.84 & $0.22-3.23$ & 0.80 \\
\hline Gastrointestinal disease & $1(3)$ & $5(5)$ & 0.61 & $0.07-5.47$ & 0.66 \\
\hline Renal pathology & $1(3)$ & $11(12)$ & 0.26 & $0.03-2.10$ & 0.21 \\
\hline Sepsis & $10(33)$ & $30(32)$ & 1.07 & $0.45-2.56$ & 0.89 \\
\hline Sepsis shock & $10(33)$ & $27(39)$ & 1.24 & $0.51-2.99$ & 0.63 \\
\hline \multicolumn{6}{|l|}{ Total SOFA score ${ }^{j}$ at ICU admission, } \\
\hline Median (range) & $6(1-18)$ & $6(0-17)$ & 1.03 & $0.93-1.14$ & 0.58 \\
\hline SAPs 3 score $^{k}$, median (range) & $66(27-97)$ & $64(29-105)$ & 1.00 & $0.98-1.02$ & 0.95 \\
\hline \multicolumn{6}{|l|}{ Invasive devices, n (\%) } \\
\hline Mechanical ventilation & $18(60)$ & $58(62)$ & 0.97 & $0.42-2.25$ & 0.95 \\
\hline Central vascular catheter & $25(83)$ & $81(86)$ & 0.80 & $0.26-2.47$ & 0.70 \\
\hline Urinary catheter & $23(77)$ & $72(76)$ & 1.00 & $0.38-2.65$ & 0.99 \\
\hline \multicolumn{6}{|l|}{ Previous use of antimicrobials, $\mathrm{n}(\%)$} \\
\hline Aminoglycosides' & $2(7)$ & $7(7)$ & 0.89 & $0.17-4.52$ & 0.89 \\
\hline Cephalosporins, $3 r d$ and $4 r d$ generations $^{m}$ & $5(17)$ & $16(17)$ & 0.98 & $0.32-2.93$ & 0.96 \\
\hline Carbapenems $^{n}$ & $5(17)$ & $32(34)$ & 0.39 & $0.14-1.11$ & 0.08 \\
\hline Glycopeptides ${ }^{\circ}$, linezolid and tigecycline & $7(23)$ & $35(37)$ & 0.51 & $0.19-1.30$ & 0.17 \\
\hline
\end{tabular}


Table 2 Clinical characteristic investigated for association with CS-GNB sepsis (Case group 2) in an adult clinical-surgical ICU (Continued)

\begin{tabular}{|c|c|c|c|c|c|}
\hline \multirow[t]{2}{*}{ Predictive Factors } & \multicolumn{2}{|l|}{ Univariate analysis } & \multirow[t]{2}{*}{ Odds ratio } & \multirow[t]{2}{*}{$95 \% \mathrm{Cl}$} & \multirow[t]{2}{*}{$p^{t}$ valor } \\
\hline & CS-GNB $(n=30)$ & Controls $(n=94)$ & & & \\
\hline Fluoroquinolones $^{p}$ & $2(7)$ & $15(16)$ & 0.38 & $0.08-1.75$ & 0.21 \\
\hline Metronidazole & $7(23)$ & $13(14)$ & 1.90 & $0.68-5.31$ & 0.22 \\
\hline Piperacilin-tazobactam & $8(27)$ & $21(22)$ & 1.26 & $0.49-3.25$ & 0.63 \\
\hline Polymyxins ${ }^{q}$ & $3(10)$ & $9(10)$ & 1.05 & $0.27-4.16$ & 0.95 \\
\hline ATB with action for anaerobes ${ }^{r}$ & $14(47)$ & $59(63)$ & 0.52 & $0.23-1.19$ & 0.12 \\
\hline Antifungal agents ${ }^{5}$ & $3(10)$ & $13(14)$ & 0.69 & $0.18-2.61$ & 0.59 \\
\hline
\end{tabular}

Abbreviations: AIDS Acquired Immunodeficiency Syndrome, ATB antibiotic agents, CI Confidence interval, CR-GNB Carbapenem resistant Gram-negative bacilli, ICU Intensive Care Unit

aprior comorbidities or conditions to investigated sepsis episode

${ }^{b}$ Diagnosis of diabetes mellitus requiring oral or injectable hypoglycemic drug

${ }^{c}$ Creatinine clearance $<30 \mathrm{cc} / \mathrm{min}$

${ }^{\mathrm{d}}$ Required in the last 90 days

eLaboratory clinical evidence

${ }^{f}$ Prednisone $>10 \mathrm{mg}$ for more than 50 days, corticosteroid for $>7$ days or immunomodulatory agents (examples: monoclonal agents, methotrexate)

In the last 30 days

${ }^{\mathrm{h}}$ Nosocomial diarrhea (3 or more daily episodes of stool for 2 or more days)

iGranulocytes $<500$ cells $/ \mathrm{mm}^{3}$

'Sequential Organ Failure Assessment score

${ }^{\mathrm{k}}$ Simplified Acute Physiology Score III

Amikacin and gentamicin

${ }^{\mathrm{m}}$ Ceftriaxone, ceftazidime and cefepime

${ }^{n}$ Ertapenem, imipenem-cilastatin and meropenem

${ }^{\circ}$ Daptomycin, teicoplanin and vancomycin

${ }^{\mathrm{P} C i p r o f l o x a c i n}$, levofloxacin, moxifloxacin

${ }^{\mathrm{q} P o l y m y x i n} \mathrm{~B}$ and colistin

'Antibacterial agents with action for anaerobes - Amoxicilin-clavulanate, ampicillin-sulbactam, piperacilin-tazobactam, clindamycin, ertapenem, imipenem, meropenem and metronidazole

${ }^{5}$ Amphotericin B family (standard, lipid complex or liposomal Amphotericin), echinocandins and azoles

tPearson's chi-square test or Fisher's exact test or Mann-Whitney-Wilcoxon $U$ test, as required, and considering statistically significant $p<0.05$

susceptibility testing of any culture isolate were performed by VITEK 2 (BioMérieux, Hazelwood, MO, USA) system, and confirmed by disk diffusion or E-test, according to the updated recommendations of Clinical and Laboratory Standards Institute [25] and European Committee on Antimicrobial Susceptibility Testing [26], including the use of meropenem, imipenem and ertapenem for all Gram-negative bacterial species, except Stenotrophomonas maltophilia which is naturally resistant to carbapenems. Carbapenemase production was investigated by phenotypic tests with phenylboronic acid and ethylene diamine tetra acetic acid.

Gram-negative bacterial isolates detected in blood and other cultures were referred to Laboratório de Pesquisa em Infecção Hospitalar for microbiological confirmation by using classical and molecular biochemical methods as described in previous publications [27, 28]. The search for the following genes of carbapenemases of Amber class A $\left(b l a_{\mathrm{KPC}-2}\right), \mathrm{B}\left(b l a_{\mathrm{SPM}-1}, b l a_{\mathrm{NDM}-1}, b l a_{\mathrm{VIM}}\right)$ and D (bla $a_{\text {OXA-23-like, }}, b l a_{\text {OXA-48-like }}$ and $\left.b l a_{\text {OXA-51-like }}\right)$ was performed by using in-house multiplex polymerase chain reaction (PCR) test.

We classified the antimicrobial susceptibility profile of the strains in multi-drug (MDR), extensively-drug (XDR) and pan-drug resistant (PDR) and described as "possible" profiles whenever not all antimicrobials of all selective classes for each bacterial group or species were tested, according to Magiorakos et al. (2012) [29]. S. maltophilia and Burkholderia cepacia were considered MDR.

\section{Sample size and statistical analysis}

Considering an alpha error of $5 \%$, a power of $80 \%$, a control to case ratio of $1: 1$, and respectively 40 and $18 \%$ exposure to carbapenem among cases and controls [19], the sample size estimated was 152 patients.

The findings were used to build a model with clinicalepidemiological factors that can be easily identified by physicians during the first moment of patient evaluation, at a time when only clinical-epidemiological parameters can guide empirical antimicrobial therapy. All variables were analyzed using SPSS $^{\circ}$ statistics v22.0 software. Categorical variables were compared using Chi-Square or Fisher's exact test and for continuous variables, the MannWhitney-Wilcoxon test was used. Collinearity was investigated initially using Pearson correlation matrix and cross-tabulations between two or more variables [30]. All variables investigated as predictors were explored in univariate and multivariate logistic regression analyses using the complete data set to identify independent risk factors for CR-GNB and CS-GNB sepsis [31]. 
To optimize the model, we used our best knowledge not to include variables with collinearity together and give chances to those clinically meaningful. Our database has many variables that may be related to our outcome and correlated with each other. Although all variables were considered, those with small frequency and those with collinearity had to be excluded from multivariate analysis to improve the fit of the model. Possible interactions were also investigated. Using the approach described above, different models were evaluated but the best-fit model came with backward selection procedure. We also have used robust fit criteria for model comparisons (AIC and BIC) [32]. Both sides of the curve and significance level of $5 \%$ were considered in all tests.

\section{Results}

Study population and clinical characteristics

Among the total of 629 ICU admissions followed by 7797 patient-days, we evaluated 342 episodes of SIRS/sepsis detected (Fig. 1). After applying the exclusion criteria, we enrolled a total of 184 patients: 60 patients who acquired CR-GNB sepsis, 30 patients with CS-GNB sepsis, and each group was compared with 94 patients with undetermined $(n=78)$ or non GNB $(n=16)$ sepsis. Demographic and

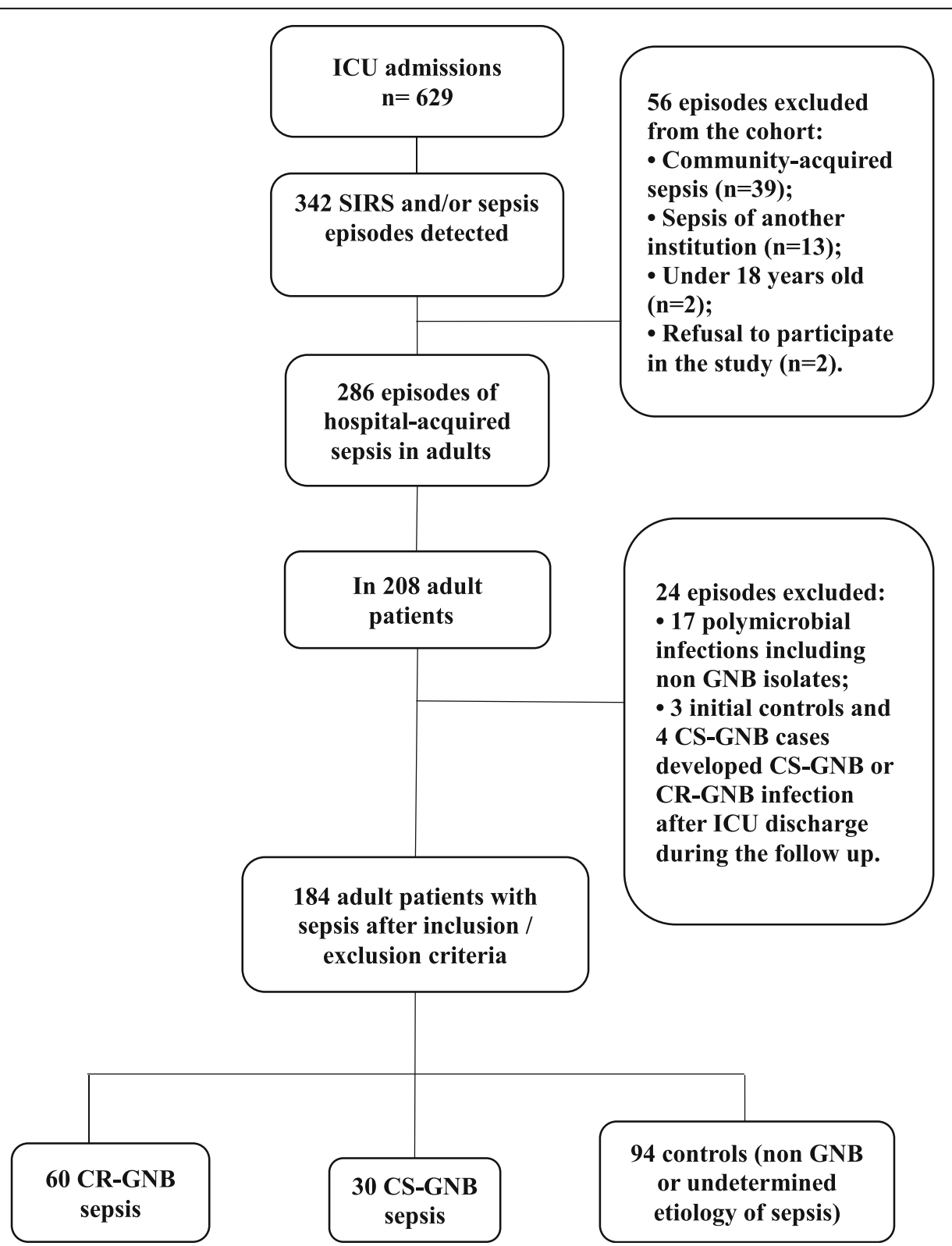

Fig. 1 Flowchart of patients included in the study. CR-GNB carbapenem-resistant Gram-negative bacilli; CS-GNB carbapenem-susceptible Gram-negative bacilli 
clinical characteristics of included patients are shown in Tables 1 and 2. Ninety-seven percent (29/30) of CS-GNB cases had severe sepsis-2, as well as $85 \%$ (51/60) of CRGNB and $84 \%(79 / 94)$ of the controls. While 94\% (51/54) of CR-GNB sepsis, $100 \%$ of CS-GNB cases (27/27) and 87\% $(74 / 85)$ of control group that could have their episode evaluable $(166 / 184,90 \%)$ fulfilled sepsis-3 criteria. Eighteen patients $(10 \%, 18 / 184)$ were considered not evaluable for the modified sepsis-3 criteria for being on mechanical ventilation and sedation. Although these patients did not have delta SOFA $\geq 2$, they had high median SOFA score at ICU admission (5.5, range 0-15) and on the date of initial blood culture collection (4, range 0-15). Septic shock was detected in $72 \%(43 / 60)$ of CR-GNB sepsis, $70 \%(21 / 30)$ of CS-GNB and 64\% (60/94) of control patients with 30-day all-cause mortality rate of $50 \%(30 / 60), 40 \%(12 / 30)$ and 45\% (42/94), respectively.

The plausibility of infectious source was categorized as definitive or probable in $89 \%(25 / 28)$ and $84 \%(54 / 64)$ of CS-GNB and CR-GNB sepsis, respectively, while the sources of infection were determined in $64 \%$ (60/94) of controls. Bacteremia was identified in 25\% (46/184) of all studied patients, and 43\% (20/46) of them were diagnosed as probable catheter related bloodstream infections (BSI).

CR-GNB cases were followed for the median of 47 days (range 1-351 days), while CS-GNB cases and controls for

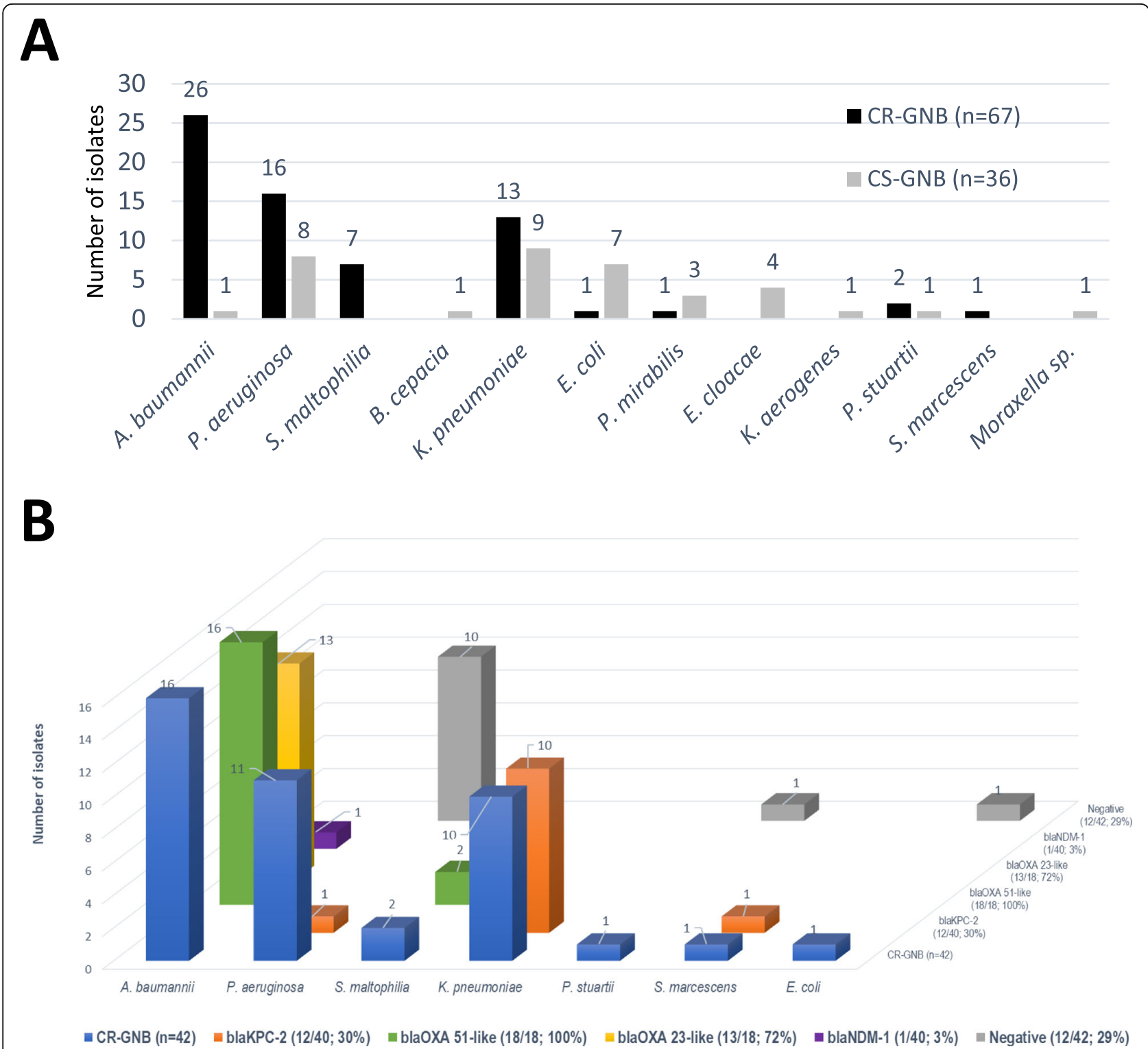

Fig. 2 a Distribution of the etiological agents of sepsis by CR-GNB $(n=67)$ and CS-GNB $(n=36)$; $\mathbf{b}$ CR-GNB isolates tested $(n=42)$ and respective carbapenemase-producing genes detected $(n=44)$. CR-GNB, carbapenem resistant Gram-negative bacilli; CS-GNB, carbapenem-susceptible Gram-negative bacilli 
40.5 and 37 days (range 1-71 days in both groups), respectively, during ICU stay until the end of follow-up period. The median length of ICU stays prior to the sepsis episode investigated was 10 times higher among CR-GNB cases (10 days, range 0-291) than CS-GNB cases (1-day, range $0-34)(p=0.006)$. Half of sepsis episodes were detected at ICU admission. Sepsis before $48 \mathrm{~h}$ of ICU hospitalization occurred in $67 \%(20 / 30)$ of CS-GNB cases group, similar to the controls $(57 \%, 54 / 94)$, and was statistically different among CR-GNB cases $(20 \%, 12 / 60)(\mathrm{OR}=$ 8.0; $95 \%$ CI $3.0-21.5 ; p<0.001)$. Therefore, the majority of later sepsis was caused by CR-GNB.

Non-fermenting bacilli corresponded to 73\% (49/67) of the etiology of CR-GNB sepsis, and A. baumannii accounted for $43 \%$ of the occurrences (26/60) (Fig. 2a). CR-GNB isolates were detected in tracheal aspirate or bronchoalveolar lavage (BAL) $(59 \%, 37 / 63)$, blood (35\%, $22 / 63)$, urine $(3 \%, 2 / 63)$ and operative wound samples $(3 \%, 2 / 63)$. The majority of isolates $(83 \%, 45 / 54)$ had MIC $\geq 16 \mathrm{mg} / \mathrm{ml}$ for meropenem and/or imipenem. Two isolates of Klebsiella pneumoniae had MIC $\geq 16 \mathrm{mg} / \mathrm{ml}$ for polymyxins and $8 \%(4 / 52)$ of strains belonged to polymyxins naturally resistant species. CR-GNB strains causing sepsis were $89 \%$ MDR (48/54), 69\% (37/54) possible extensively drug-resistant and $2 \%(1 / 54)$ had possible pandrug-resistant profile. The production of carbapenemases was estimated in 77\% (39/51) of the isolates tested by phenotypic tests $(92 \%, 12 / 13)$ and/or by PCR technique $(74 \%, 35 / 47)$, since we included tested $(n=2)$ but also non-tested S. maltophilia isolates $(n=5)$.

Figure $2 \mathrm{~b}$ shows the CR-GNB isolates investigated $(n=42)$ and respective carbapenemase-producing genes detected $(n=44)$. Species of the Enterobacteriaceae family $(69 \%$; 25/36) outweighed among the etiologies of CSGNB sepsis (Fig. 2a). The isolates were recovered from blood $(33 \%, 11 / 33)$, tracheal aspirate or BAL $(30 \%, 10 /$ $33)$, operative wound samples $(21 \%, 7 / 33)$ and urine $(15 \%, 5 / 33)$. The susceptibility profiles of the isolates were $79 \%(27 / 34)$ non-MDR and $21 \%(7 / 34)$ classified as MDR. Polymyxin intrinsic resistance was detected in $13 \%(4 / 31)$ of CS-GNB isolates.

Ventilator-associated pneumonia (VAP) was the most frequent infectious source among CR-GNB sepsis (48\%; $32 / 66$ ) with the predominance of $A$. baumannii in $56 \%$
(18/32) of etiological agents. Among CS-GNB sepsis, respiratory tract infections (VAP, hospital-acquired pneumonia and tracheobronchitis) occurred in 37\% (11/30). Intra-abdominal surgical site (SSI) plus urinary-tract (UTI) infections prevailed in CS-GNB sepsis (43\%; 13/ $30)$ than CR-GNB sepsis $(11 \%$; $7 / 66)(p<0.001)$. While in the control patients, excluding cases with undetermined focus $(36 \%, 34 / 94)$, hospital-acquired pneumonia $(32 \%, 21 / 66)$ and surgical wound $(32 \%, 21 / 66)$ predominated as source of sepsis.

\section{CR-GNB septic patients versus control patients}

The results of comparative univariate analysis between cases of CR-GNB sepsis versus controls are shown in Table 1. Previous infection was an important factor associated with CR-GNB sepsis (77\%; 46/60) when compared to control group, with high percentage of healthcareassociated infection $(57 \% ; 26 / 46)$ and $48 \%(22 / 46)$ of previous sepsis at the same hospitalization period. Previous infection was predominantly of bacterial origin among CR-GNB cases $(50 \%$; $30 / 60)$ than controls $(27 \%$; $25 / 94)(\mathrm{OR}=2.74 ; 95 \%$ CI $1.39-5.48 ; p=0.004)$. Sepsis as a reason for ICU admission occurred in $52 \%(31 / 60)$ of the CR-GNB cases, contrasting with only $32 \%(30 / 94)$ of controls $(\mathrm{OR}=2.27 ; 95 \%$ CI 1.16-4.46; $p=0.016)$. Sepsis recurred during ICU stay in $65 \%(39 / 60)$ of CRGNB cases and only $19 \%(18 / 94)$ of controls $(\mathrm{OR}=7.71$; 95\% CI 3.72-16.51; $p<0.0001)$.

There was no difference between cases (77\%; 46/60) versus controls $(79 \%$; 74/94) in respect to the use of antibiotics during the 3 days prior to the collection of blood culture $(p=0.76)$, including antibiotics active on GNB ( $70 \%$ vs. $73 \% ; p=0.64)$ or polymyxins $(28 \%$ vs. $18 \% ; p=0.13)$ were used. However, there was a difference in carbapenem consumption among these groups $(36 / 60,60 \%$ vs. $39 / 94,41 \%$; $\mathrm{OR}=2.11 ; 95 \%$ CI 1.09 $4.12 ; p=0.026)$.

The independent risk factors for CR-GNB sepsis are previous infection (mostly hospital-acquired bacterial infection or sepsis) $(\mathrm{OR}=4.28 ; 95 \%$ CI $1.77-10.35 ; p=$ $0.001)$, previous use of mechanical ventilation $(\mathrm{OR}=$ $4.21 ; 95 \%$ CI $1.17-15.18 ; p=0.028)$ and carbapenems $(\mathrm{OR}=3.42 ; 95 \%$ CI $1.37-8.52 ; p=0.008)$, and length of

Table 3 Independent predictive factors associated with CR-GNB sepsis; multivariate logistic regression

\begin{tabular}{|c|c|c|c|c|c|}
\hline Risk factors for sepsis by CR-GNB & CR-GNB cases $(n=60)$ & Control group $(n=94)$ & Odds ratio & $95 \% \mathrm{Cl}$ & $p^{\text {a }}$ value \\
\hline Previous infection, n (\%) & $46(77)$ & $31(33)$ & 4.28 & $1.77-10.35$ & 0.001 \\
\hline Mechanical ventilation, $\mathrm{n}(\%)$ & $56(93)$ & $58(62)$ & 4.21 & $1.17-15.18$ & 0.028 \\
\hline Use of carbapenem, n (\%) & $48(80)$ & $32(34)$ & 3.42 & $1.37-8.52$ & 0.008 \\
\hline Length of hospital stay (days), median (range) & $26(1-375)$ & $10(0-143)$ & 1.03 & $1.01-1.05$ & 0.007 \\
\hline
\end{tabular}

Abbreviations: CR-GNB carbapenem-resistant Gram-negative bacilli, $\mathrm{Cl}$ confidence interval

${ }^{a}$ Wald test for logistic regression, $p$ significant $<0.05$, accuracy of $80 \%$ for CR-GNB model 
hospital stay $(\mathrm{OR}=1.03 ; 95 \%$ CI $1.01-1.05 ; p=0.007)$ (Table 3).

\section{CS-GNB septic versus control patients}

Table 2 shows the comparative univariate analysis between cases of CS-GNB sepsis versus controls. Repeated episodes of sepsis occurred during ICU stay in 23\% (7/30) of CS-GNB cases with no significance when compared to controls $(p=0.619)$. Regarding the use of antimicrobial agents during the 3 days prior to the initial blood culture that could interfere with its result, there was statistically significant difference between CS-GNB sepsis (53\%; 16/ $30)$ and controls (79\%; 74/94) (OR $=0.31 ; 95 \%$ CI 0.13 , $0.75 ; p=0.009)$. The same occurred with the use of carbapenems $(3 / 30,10 \%$ vs. $39 / 94,41 \%$; OR $=0.16 ; 95 \% \mathrm{CI}$ $0.03-0.57 ; p=0.0009)$. However, patients with CS-GNB sepsis did not receive polymyxins previously, but control patients received them $(18 \% ; 17 / 94)$.

In multivariate analysis, readmission to the ICU (OR = 6.92; 95\% CI 1.72-27.78; $p=0.006)$ and prior nosocomial diarrhea $(\mathrm{OR}=5.32$; 95\% CI 1.07-26.45; $p=0.041)$ were detected as independent risk factors for developing CS-GNB sepsis in the study population.

\section{Discussion}

In this study, patients with longer length of hospital stay, previous infection, mostly hospital acquired bacterial infection, who had been previously treated with mechanical ventilation and carbapenems presented a higher risk of sepsis due to CR-GNB than control group. Whereas readmission to the $\mathrm{ICU}$ and prior nosocomial diarrhea were factors associated with CS-GNB sepsis in the study population.

Previous infection has been rarely reported as risk for infection/colonization or bacteremia by CR A. baumannii $[7,8]$. In our cohort, previous infection was the most important factor for CR-GNB sepsis, mainly of bacterial origin, mostly nosocomial infection and $48 \%$ previous sepsis. Recurrent pattern of sepsis was a striking feature of our studied population. Therefore, more attention for the prevention and control of nosocomial sepsis is required for the prevention of subsequent hospital-acquired CR-GNB sepsis, especially caused by $A$. baumannii, at the same hospitalization period in ICU patients.

The studies that have evaluated risk factors for CRGNB or CR A. baumannii bacteremia, SIRS or nosocomial infection have found mechanical ventilation, respiratory failure, but also VAP as risk factors in critically ill patients $[7,8,18,19]$. In our study we did not investigate types of infection as predictive factors because we sought to investigate variables that would readily discriminate patients with increased risk, in order to be useful to guide empirical therapy in future.
Prolonged hospital stay is a classically recognized risk for hospital and ICU infection [10, 11, 14, 19, $33]$. We found a $2 \%$ increase in the chances of developing sepsis by CR-GNB for every day of hospitalization. ICU readmission is also a documented risk factor for the acquisition of CS-GNB infection $[10,16]$. A few studies have found diarrhea to be associated with GNB bacteremia [34-36].

As far as we know, this is the first risk factor study in CR-GNB sepsis that has considered repetitive episodes and not only bacteremia, but the broad variety of infection sources commonly observed in ICU sepsis. Studies that have investigated risk factors for infections by $\mathrm{CR}$ GNB species are still rare $[18,19]$. In general, the studies focus on risk factors for infections by specific species such as $K$. pneumoniae $[12,13,16]$ or other members of the Enterobacteriaceae $[15,17], P$. aeruginosa $[10,11]$ and A. baumannii [7-9], and most of them in hospital infection $[9,10,12,15-17]$ or bacteremia $[8,11,18,19]$. However, bacteremia has been detected in less than $30 \%$ of septic cases in ICU $[37,38]$. The extensive use of vascular catheter has been recognized as the most important factor contributing to BSI [4, 39, 40], while VAP has predominated as source of sepsis in ICU [38]. Therefore, although bacteremia can be considered the gold standard it represents only part of the population who were diagnosed with sepsis in ICU and is frequently associated with vascular catheter infection. In addition, several risk factor studies select the first episode of infection or bacteremia only [8-10, 13, 16-19]. The successful longitudinal follow-up of this cohort study allowed identifying patients who presented recurrent sepsis, whose diagnoses were also essential to better select cases and controls. Giving the chance of inclusion of all episodes and the variety of sepsis infections may be more reliable to better discriminate patients at higher risk in ICU.

The case-case-control design is more effective for the identification of risk factors for antimicrobial resistant pathogens, avoiding bias of exposure to the antimicrobial of interest, for not using CS-GNB sepsis as control group $[41,42]$. Our study aimed to confirm exposure to carbapenems as a risk for the development of CR-GNB sepsis $[11,12,18,19,43]$, since our control group was composed of patients with sepsis and antimicrobial treatment. This methodology allows the application of the factors found in the management of empirical therapy, which would not be possible if the control was a patient without sepsis and antimicrobial treatment of the episode investigated [42].

The design resulted in the selection of cases so efficiently that the study detected a remarkable difference in the etiologies of sepsis by CR-GNB and CS-GNB. Indeed, the CR-GNB sepsis presented a predominance of non-fermenting bacteria, mainly $A$. baumannii, which was likely related to the higher prevalence of respiratory 
tract infections as source of sepsis $[18,19]$. Whereas Enterobacteriaceae species predominated among CS-GNB sepsis, mostly associated with respiratory tract infections, but also intra-abdominal SSI and UTI.

Evaluated sepsis episodes in cases and control patients had a good agreement when considering the adapted parameters for the diagnosis of sepsis-3 [21]. The plausibility of infectious source as definitive and probable occurred in a high proportion in all groups of cases while the source of infection was determined in the majority of controls.

Our data characterize sepsis by CS-GNB mostly as hospital-acquired infection outside the ICU, whereas sepsis by CR-GNB was mainly ICU-acquired infection. This issue corroborates with a well-known fact that ICU patients have more risk of infection by resistant bacteria [33]. In fact, CR-GNB species typify a bacterial population with a high level of resistance and few treatment options, due to the often high carbapenems MIC and some combined resistance to polymyxins. Molecular investigation of carbapenemase production shows that we have determined the risk factors for CR-GNB sepsis predominantly with this mechanism of resistance, which is commonly described and disseminated worldwide [43-47].

Main limitation of this study may be described as a single center study, which indicates caution to any generalization of our findings. Adapting management strategies to the local epidemiological data is a general recommendation for the prevention and control of hospital-acquired infection, which indicates the performance of cyclic evaluations locally. The methodology of case-control selection was one of the study's strengths. The performance of microbiological methods and the use of antimicrobials, which may have inhibited microbial growth in clinical cultures, could have influenced the risk factors results. Other non-investigated elements may also have interfered. Phylogenetic analysis of GNB isolates would have improved our knowledge about the epidemiological context of GNB hospital-acquired sepsis.

\section{Conclusions}

Prolonged hospitalization with the development of healthcare-associated infection, requiring mechanical ventilation and treatment with carbapenems seem to be the natural history for subsequent sepsis by carbapenemase-producing GNB in this population. The concordance with the background knowledge suggests that these factors should be evaluated further for developing and validating a risk score to identify patients at higher risk for CR-GNB sepsis in ICU. Little is known about the influence of recurrent sepsis during the same hospitalization period. Consequently, investigation of repetitive episodes of sepsis in ICU patients is warranted.

\section{Supplementary information}

Supplementary information accompanies this paper at https://doi.org/10. 1186/s13756-020-00791-w.

Additional file 1 Appendix S1. Study Form 1, with variables investigated in this study. Appendix S2. Study Form 2, with variables investigated in this study. Appendix S3. Study Form 2a, with variables investigated in this study. Appendix S4. STROBE Statement-Checklist of items included in this study.

\section{Abbreviations}

BAL: Broncho alveolar lavage; BSI: Bloodstream infection; CS: Carbapenemsusceptible; CR: Carbapenem-resistant; GNB: Gram-negative bacilli; ICU: Intensive care units; MIC: Minimum Inhibitory Concentration; MDR: Multidrug resistant; PCR: Polymerase chain reaction; SAPS-3: Simplified Acute Physiology Score III; SIRS: Systemic Inflammatory Response Syndrome; SSI: Surgical site infection; SOFA: Sequential Organ Failure Assessment; UTI: Urinary-tract infection; VAP: Ventilator-Associated Pneumonia; XDR: Extensively drug resistant; PDR: Pan-drug resistant

\section{Acknowledgements}

Nucleus of Hospital Research collaborators: Glória Regina A. A. Magalhães², Priscila P. Silva ${ }^{3}$, Scyla Maria S. A. S. Reis Di Chiara², Amanda Aparecida S. Machado ${ }^{3}$, Thaisa M. Tozo ${ }^{2}$, Lucas Lameirão P. A. Rosas ${ }^{3}$, Paulo C. M. Barros², Wania V. de Freitas ${ }^{2}$, Ana Paula D. C. Assef ${ }^{1}$. The research leading to these results is supported by the Technical Cooperation Agreement (number 51/2017) between FIOCRUZ and HFSE to develop research.

Email address of study collaborators: gramagal@hotmail.com, priscila. pinho@ioc.fiocruz.br, scylareiss@gmail.com, amandacidamachado@gmail.com, thaisa.mdrs@gmail.com, lucas.rosas40@gmail.com, paulocesar.unirio@outlook. com, freitas.wania@gmail.com, anapdca@ioc.fiocruz.br

\section{Authors' contributions}

Supervised the study: MZRG Designed the analytic plan: MZRG, SCSG Obtained scholarships: MZRG Enrolled patients: MZRG, EML Collected data: EML, PAC, DSB, LHZP, JPST, NDL, CSB Created and managed the database: MZOA, MZRG Analyzed and/or interpreted the data: SCSG, MZRG, EML, CASR, MDA, RQS, JAM, APDCA Acted as supervisor for data analysis and/or interpretation: MZRG. Drafted the initial manuscript: EM L Reviewed and/or revised the manuscript: MZRG, SCSG. All authors read and approved the final manuscript.

\section{Funding}

Research Support Foundation of the State of Rio de Janeiro (FAPERJ) and National Council for Scientific and Technological Development (CNPq) for providing scholarships for scientific initiation for undergraduate medical students.

\section{Availability of data and materials}

The datasets used and/or analysed during the current study are available from the corresponding author on reasonable request.

Ethics approval and consent to participate

This study was approved by FIOCRUZ and HFSE Ethics Committees (CAAE: 39978114.5.0000.5248 and CAAE 39978114.5.3002.5252).

Consent for publication

Not applicable.

\section{Competing interests}

The authors declare that they have no competing interests

\section{Author details}

${ }^{1}$ Laboratório de Genética Molecular de Microrganismos, Instituto Oswaldo Cruz, Oswaldo Cruz Foundation (IOC/FIOCRUZ), Rio de Janeiro, RJ, Brazil. 'Laboratório de Pesquisa em Infecção Hospitalar, Instituto Oswaldo Cruz, Oswaldo Cruz Foundation (IOC/FIOCRUZ), Rio de Janeiro, RJ, Brazil. ${ }^{3}$ Hospital Federal dos Servidores do Estado (HFSE), Ministry of Health, Rio de Janeiro, RJ, Brazil. ${ }^{4}$ Instituto Fernandes Figueira, Oswaldo Cruz Foundation, Rio de Janeiro, RJ, Brazil. 
Received: 17 April 2019 Accepted: 26 July 2020

Published online: 14 August 2020

\section{References}

1. Braber A, van Zanten AR. Unravelling post-ICU mortality: predictors and causes of death. Eur J Anaesthesiol. 2010;27:486-90.

2. Prowle JR, Echeverri JE, Ligabo EV, Sherry N, Taori GC, Crozier TM, et al. Acquired bloodstream infection in the intensive care unit: incidence and attributable mortality. Crit Care. 2011;15:R100.

3. Gaynes R, Edwards JR. National Nosocomial Infections Surveillance S. Overview of nosocomial infections caused by gram-negative bacilli. Clin Infect Dis. 2005;41:848-54.

4. Tabah A, Koulenti D, Laupland K, Misset B, Valles J, de Bruzzi CF, et al. Characteristics and determinants of outcome of hospital-acquired bloodstream infections in intensive care units: the EUROBACT. Intensive Care Med. 2012;38:1930-45.

5. Luna CM, Rodriguez-Noriega E, Bavestrello L, Guzman-Blanco M. Gramnegative infections in adult intensive care units of latin america and the Caribbean. Crit Care Res Pract. 2014. Article ID 480463, 12 pages. http://dx. doi.org/10.1155/2014/480463.

6. Kousouli E, Zarkotou O, Politi L, Polimeri K, Vrioni G, Themeli-Digalaki K, et al. Infection control interventions affected by resource shortages: impact on the incidence of bacteremias caused by carbapenem-resistant pathogens. Eur J Clin Microbiol Infect Dis. 2018;37:43-50.

7. Romanelli RM, Jesus LA, Clemente WT, Lima SS, Rezende EM, Coutinho RL, et al. Outbreak of resistant Acinetobacter baumannii- measures and proposal for prevention and control. Braz J Infect Dis. 2009;13:341-7.

8. Routsi C, Pratikaki M, Platsouka E, Sotiropoulou C, Nanas S, Markaki V, et al. Carbapenem-resistant versus carbapenem-susceptible Acinetobacter baumannii bacteremia in a Greek intensive care unit: risk factors, clinical features and outcomes. Infection. 2010;38:173-80.

9. Sheng WH, Liao CH, Lauderdale TL, Ko WC, Chen YS, Liu JW, et al. A multicenter study of risk factors and outcome of hospitalized patients with infections due to carbapenem-resistant Acinetobacter baumannii. Int J Infect Dis. 2010;14:e764-9.

10. Gomes MZ, Oliveira RV, Machado CR, Conceicao Mde S, Souza CV, Lourenco MC, et al. Factors associated with epidemic multiresistant Pseudomonas aeruginosa infections in a hospital with AIDS-predominant admissions. Braz J Infect Dis. 2012;16:219-25.

11. Dantas RC, Ferreira ML, Gontijo-Filho PP, Ribas RM. Pseudomonas aeruginosa bacteraemia: independent risk factors for mortality and impact of resistance on outcome. J Med Microbiol. 2014;63:1679-87.

12. Gomez Rueda V, Zuleta Tobon JJ. Risk factors for infection with carbapenem-resistant Klebsiella pneumoniae: a case-case-control study. Colomb Med (Cali). 2014:45:54-60.

13. Kofteridis DP, Valachis A, Dimopoulou D, Maraki S, Christidou A, Mantadakis E, et al. Risk factors for carbapenem-resistant Klebsiella pneumoniae infection/colonization: a case-case-control study. J Infect Chemother. 2014; 20:293-7.

14. Ozgur ES, Horasan ES, Karaca K, Ersoz G, Nayci Atis S, Kaya A. Ventilatorassociated pneumonia due to extensive drug-resistant Acinetobacter baumannii: risk factors, clinical features, and outcomes. Am J Infect Control. 2014;42:206-8

15. de Jager P, Chirwa T, Naidoo S, Perovic O, Thomas J. Nosocomial outbreak of New Delhi Metallo-beta-Lactamase-1-producing gram-negative bacteria in South Africa: a case-control study. PLoS One. 2015;10:e0123337.

16. Candevir Ulu A, Kurtaran B, Inal AS, Komur S, Kibar F, Yapici Cicekdemir H, et al. Risk factors of carbapenem-resistant Klebsiella pneumoniae infection: a serious threat in ICUs. Med Sci Monit. 2015:21:219-24.

17. Wang Q, Zhang Y, Yao X, Xian H, Liu Y, Li H, et al. Risk factors and clinical outcomes for carbapenem-resistant Enterobacteriaceae nosocomial infections. Eur J Clin Microbiol Infect Dis. 2016;35:1679-89.

18. Routsi C, Pratikaki M, Platsouka E, Sotiropoulou C, Papas V, Pitsiolis T, et al. Risk factors for carbapenem-resistant gram-negative bacteremia in intensive care unit patients. Intensive Care Med. 2013:39:1253-61

19. Ting SW, Lee CH, Liu JW. Risk factors and outcomes for the acquisition of carbapenem-resistant gram-negative bacillus bacteremia: a retrospective propensity-matched case control study. J Microbiol Immunol Infect. 2018;51:621-8.

20. von Elm E, Altman DG, Egger M, Pocock SJ, Gotzsche PC, Vandenbroucke $J P$, et al. The strengthening the reporting of observational studies in epidemiology (STROBE) statement: guidelines for reporting observational studies. PLoS Med. 2007:4:e296.

21. Singer M, Deutschman CS, Seymour CW, Shankar-Hari M, Annane D, Bauer $M$, et al. The third international consensus definitions for Sepsis and septic shock (Sepsis-3). JAMA. 2016;315:801-10.

22. Levy MM, Fink MP, Marshall JC, Abraham E, Angus D, Cook D, et al. 2001 SCCM/ESICM/ACCP/ATS/SIS international Sepsis definitions conference. Crit Care Med. 2003;31:1250-6.

23. Klein Klouwenberg PM, Ong DS, Bos LD, de Beer FM, van Hooijdonk RT, Huson MA, et al. Interobserver agreement of Centers for Disease Control and Prevention criteria for classifying infections in critically ill patients. Crit Care Med. 2013;41:2373-8.

24. CDC. National Healthcare Safety Network (NHSN) Patient Safety Component Manual. 2017: https://www.cdc.gov/nhsn/pdfs/pscmanual/pcsmanual_ current.pdf. Accessed 16 June 2018.

25. CLSI. Twentieth eighth informational supplement. CLSI document M100Ed28. Wayne,PA: Clinical laboratory Standards Institute; 2018. Accessed 16 June 2018.

26. EUCAST. European Committee on Antimicrobial Susceptibility Testing Breakpoint tables for interpretation of MICs and zone diameters. Version 8.1, valid from 2018-05-15 http://www.eucast.org/clinical_breakpoints/. Accessed 16 June 2018.

27. Carvalho KR, Carvalho-Assef AP, Peirano G, Santos LC, Pereira MJ, Asensi MD. Dissemination of multidrug-resistant Acinetobacter baumannii genotypes carrying Bla (OXA-23) collected from hospitals in Rio de Janeiro, Brazil. Int J Antimicrob Agents. 2009;34:25-8.

28. Seki LM, Pereira PS, de Souza MP, Conceicao Mde S, Marques EA, Porto CO, et al. Molecular epidemiology of KPC-2- producing Klebsiella pneumoniae isolates in Brazil: the predominance of sequence type 437. Diagn Microbiol Infect Dis. 2011;70:274-7.

29. Magiorakos AP, Srinivasan A, Carey RB, Carmeli Y, Falagas ME, Giske CG, et al. Multidrug-resistant, extensively drug-resistant and pandrug-resistant bacteria: an international expert proposal for interim standard definitions for acquired resistance. Clin Microbiol Infect. 2012;18:268-81.

30. Rosner BR. Fundamentals of Biostatistics. 8th ed. Mason: Cengage Learning; 2015.

31. Hosmer DW, Lemeshow S, Sturdivant RX. Applied logistic regression. 3rd ed. New York: Wiley; 2013.

32. Henley SS, Golden RM, Kashner TM. Statistical modeling methods: challenges and strategies. Biostatistics Epidemiol. 2019:1-35.

33. Fish DN, Ohlinger MJ. Antimicrobial resistance: factors and outcomes. Crit Care Clin. 2006;22:291-311.

34. Amit S, Mishali H, Kotlovsky T, Schwaber MJ, Carmeli Y. Bloodstream infections among carriers of carbapenem-resistant Klebsiella pneumoniae: etiology, incidence and predictors. Clin Microbiol Infect. 2015;21:30-4.

35. Satlin MJ, Cohen N, Ma KC, Gedrimaite Z, Soave R, Askin G, et al. Bacteremia due to carbapenem-resistant Enterobacteriaceae in neutropenic patients with hematologic malignancies. J Inf Secur. 2016;73:336-45.

36. Falcone M, Russo A, Iraci F, Carfagna P, Goldoni P, Vullo V, et al. Risk factors and outcomes for bloodstream infections secondary to Clostridium difficile infection. Antimicrob Agents Chemother. 2016;60:252-7.

37. Seymour CW, Liu VX, Iwashyna TJ, Brunkhorst FM, Rea TD, Scherag A, et al. Assessment of clinical criteria for sepsis: for the third international consensus definitions for Sepsis and septic shock (Sepsis-3). Jama. 2016;315: 762-74.

38. Giamarellos-Bourboulis EJ, Tsaganos T, Tsangaris I, Lada M, Routsi C, Sinapidis $D$, et al. Validation of the new Sepsis-3 definitions: proposal for improvement in early risk identification. Clin Microbiol Infect. 2017;23:104-9.

39. Timsit JF, Soubirou JF, Voiriot G, Chemam S, Neuville M, Mourvillier B, et al. Treatment of bloodstream infections in ICUs. BMC Infect Dis. 2014;14:489.

40. Bassetti $M$, Righi $E$, Carnelutti A. Bloodstream infections in the intensive care unit. Virulence. 2016;7:267-79.

41. Harris AD, Karchmer TB, Carmeli Y, Samore MH. Methodological principles of case-control studies that analyzed risk factors for antibiotic resistance: a systematic review. Clin Infect Dis. 2001;32:1055-61.

42. Kaye KS, Harris AD, Samore M, Carmeli Y. The case-case-control study design: addressing the limitations of risk factor studies for antimicrobial resistance. Infect Control Hosp Epidemiol. 2005;26:346-51.

43. Hotta G, Matsumura Y, Kato K, Nakano S, Yunoki T, Yamamoto M, et al. Risk factors and outcomes of Stenotrophomonas maltophilia bacteraemia: a 
comparison with bacteraemia caused by Pseudomonas aeruginosa and Acinetobacter species. PLoS One. 2014;9:e112208.

44. Queenan AM, Bush K. Carbapenemases: the versatile beta-lactamases. Clin Microbiol Rev. 2007;20:440-58 table of contents.

45. Zavascki AP, Carvalhaes CG, Picao RC, Gales AC. Multidrug-resistant

Pseudomonas aeruginosa and Acinetobacter baumannii: resistance mechanisms and implications for therapy. Expert Rev Anti-Infect Ther. 2010;8:71-93.

46. Nordmann P, Naas T, Poirel L. Global spread of Carbapenemase-producing Enterobacteriaceae. Emerg Infect Dis. 2011;17:1791-8.

47. Bush K. Carbapenemases: partners in crime. J Glob Antimicrob Resist. 2013;1: 7-16.

\section{Publisher's Note}

Springer Nature remains neutral with regard to jurisdictional claims in published maps and institutional affiliations.

Ready to submit your research? Choose BMC and benefit from:

- fast, convenient online submission

- thorough peer review by experienced researchers in your field

- rapid publication on acceptance

- support for research data, including large and complex data types

- gold Open Access which fosters wider collaboration and increased citations

- maximum visibility for your research: over $100 \mathrm{M}$ website views per year

At BMC, research is always in progress.

Learn more biomedcentral.com/submissions 